\title{
BMJ Open Systematic review of psychosocial needs assessment tools for caregivers of paediatric patients with dermatological conditions
}

\author{
Carleen Walsh (D) ,' Gerard Leavey (D) , ${ }^{1}$ Marian McLaughlin ${ }^{2}$
}

To cite: Walsh C, Leavey G, McLaughlin M. Systematic review of psychosocial needs assessment tools for caregivers of paediatric patients with dermatological conditions. BMJ Open 2022;12:e055777. doi:10.1136/ bmjopen-2021-055777

- Prepublication history and additional supplemental material for this paper are available online. To view these files, please visit the journal online (http://dx.doi.org/10.1136/ bmjopen-2021-055777).

Received 26 July 2021 Accepted 13 December 2021

Check for updates

(C) Author(s) (or their employer(s)) 2022. Re-use permitted under CC BY-NC. No commercial re-use. See rights and permissions. Published by BMJ.

${ }^{1}$ Bamford Centre for Mental Health and Wellbeing, Ulster University, Coleraine, UK

${ }^{2}$ School of Psychology, Ulster University, Coleraine, UK

Correspondence to

Carleen Walsh;

walsh-c33@ulster.ac.uk

\section{ABSTRACT}

Objective To identify validated dermatology-specific and disease-specific psychosocial needs assessment tools for caregivers of paediatric patients with dermatological conditions. A secondary objective was to assess the adequacy of their measurement properties.

Design Systematic review.

Data sources EMBASE, PsycINF0, MEDLINE (in Ovid SP), Cochrane, Cumulative Index to Nursing and Allied Health EBSCO, $U$ Search and Web of Science were searched (2000-5 October 2021). Grey literature, bibliographies, online databases of QoL tools and several trial registers were searched (2000-5 0ct 2021).

Eligibility criteria Eligible studies involved adult caregivers caring for a child (no age limit) with any form of any skin condition. Predetermined exclusion criteria, as per protocol, were applied to the search results.

Data abstraction and synthesis Title, abstract, fulltext screening and data abstraction (standardised forms) were done independently in duplicate. Both's predefined methodological criteria assessed risk of bias. Narrative synthesis was used to present the findings.

Results 187 full-text articles were examined from a total of 8979 records. Most tools were generic QoL tools, relevant to spouse/partner or based on their child's perception of the disease or assessed patients' quality of life. Following quality appraisal, 26 articles were identified, and 11 tools (1 dermatology-specific and 10 disease-specific) were included. Information outcome domains were provided for each tool (study specific, questionnaire specific, adequacy of measurement properties and risk of bias). No literature was found pertaining to the use of these tools within healthcare settings and/or as e-tools.

Discussion With limited evidence supporting the quality of their methodological and measurement properties, this review will inform future dermatological Core Outcome Set development and improve evidence-based clinical decisions. Increasing demand on limited healthcare resources justifies the codevelopment of an accessible solution-focused psychosocial needs assessment e-tool to promote caregiver health outcomes.

PROSPERO registration number PROSPERO (CRD42019159956).

\section{Strengths and limitations of this study}

- The first systematic review to provide a comprehensive overview of psychosocial assessment tools validated for use among dermatological caregivers of paediatric patients.

- This study was conducted with the involvement of a health and life subject-specific librarian and an international multidisciplinary expert group.

- The protocol was registered on the PROSPERO database (CRD42019159956), the COMET database and was conducted according to the recommendations from the Preferred Reporting Items for Systematic Reviews and Meta-Analyses 2020 statement and ENTREQ statement.

- Adequacy of measurement properties was assessed using Both et al's criteria.

- Included articles were limited to being published in English between 2000 and 2021.

\section{INTRODUCTION}

Paediatric dermatology is a unique speciality in that children with lifelong and life-limiting skin disorders are increasingly being cared for by caregivers at home, ${ }^{1}$ which requires considerable cognitive, emotional and physical resources. ${ }^{2}$ Skin disease is the fourth leading cause of global disease burden with associated prevalence, care requirements and costs comparable with other diseases, such as cardiovascular disease and diabetes. ${ }^{3-5}$ Delayed identification of dermatological caregiver needs and provision of timely supports can seriously compromise the long-term psychosocial well-being of caregivers ${ }^{6-10}$ and particularly undermine the care and treatment of paediatric patients affected by rare or chronic skin disease. ${ }^{11}$ Caregivers of skin disease require similar systems of monitoring and integrated biopsychosocial support as other comparable chronic conditions.

The WHO directive,${ }^{12}$ recent international guidelines ${ }^{1314}$ and reports ${ }^{91516}$ emphasise the importance of identifying psychosocial needs 
assessment tools for use among long-term caregivers, particularly self-referral models. Timely and appropriate identification of caregivers' unmet psychosocial needs has the potential to reduce caregiver strain and increase their ability to provide quality care within the home at reduced public health cost. Although a psychosocial needs assessment could be considered preventative in nature, by anticipating caregiver burnout and decreasing the need for emergency interventions, there is a lack of evidence regarding the use of caregiver assessment tools within healthcare settings. To date, no comprehensive review of psychosocial needs assessment tools validated for use among informal dermatological caregivers of paediatric patients has been conducted. With increasing competition for valuable healthcare resources and services, there is an urgent need to reconceptualise global burden within the construct of 'prevention is better than cure' by informing evidence-based decisions and promoting caregiver health outcomes within day-to-day clinical practice.

\section{Objectives}

This review aimed to improve clinician access to existing dermatology-specific and disease-specific psychosocial needs assessment tools, validated for use among caregivers of paediatric patients with dermatological conditions. Additionally, this review assessed the adequacy of their measurement properties.

\section{METHODS}

This review was conducted according to the recommendations from the Preferred Reporting Items for Systematic Reviews and Meta-Analyses (PRISMA) 2020 statement. ${ }^{17}$ The ENTREQ statement was read and guided in reporting the synthesis of the findings. ${ }^{18}$

\section{Eligibility criteria}

Studies that involved adult caregivers (age 18 years and over) caring for a child (no age limit) with any form of any skin condition were included. Predetermined exclusion criteria were adhered to (see protocol). Included articles were limited to being published in English between 01 January 2000 and 5 October 2021. This ensured that relevant assessment tools developed in the years before publication of the 2017 review $^{19}$ were included as that review had limited their search to one database and quality-oflife measures only, which contrasts with the measures recommended by the Cochrane Skin Centre of Evidence Based Dermatology.

\section{Information sources}

MEDLINE, PsycINFO and Embase (OVID interface) and Cumulative Index to Nursing and Allied Health Literature (CINAHL) EBSCO were searched (1 January 2000-5 October 2021). Grey literature, bibliographies, online databases of QoL tools and several trial registers were also searched (1 January 2000-5 October 2021). A 'snowball' search was carried out to identify additional studies by manually searching the reference lists of all publications eligible for full-text review. The PRISMA flow diagram (figure 1) includes the number of records identified from each source.

\section{Search strategy}

One known relevant systematic review ${ }^{19}$ was used as a starting point to identify records. A draft search strategy was developed by using candidate search terms that were identified in the titles, abstracts and subject indexing of that systematic review. The full search strategy development process is included in online supplemental file 1. This strategy was tailored to the specifications of each of the databases searched and developed in collaboration with a subject-specific librarian (JA) and expert group. Each tailored database search strategy is included in online supplemental file 2. All search terms/categories used to search within the supplementary sources are included in online supplemental file 3 .

\section{Selection and data collection process}

Title, abstract and full-text screening were conducted manually in duplicate (independently) by two reviewers (CW and GL). Extracted data from full-text articles was processed using three standardised extraction forms: (1) study-specific information included the name of the tool, country of origin, disease of affected patients, sample sizes used in each stage of its development and study setting; (2) questionnaire-specific information included the outcome domains, number of items and subscales, recall period, scoring system, respondent feedback and administration mode and time; (3) adequacy of measurement properties was evaluated using five methodological domains: validity, reliability, structure, interpretability and transferability. At the full-text screening stage, any discrepancies were resolved by discussion and, where necessary, the third author (MM) was consulted.

\section{Risk of bias assessment}

Risk of bias in the included studies was assessed independently by two reviewers using Both et al's ${ }^{20}$ criteria, made possible by the similarities between the studies. Each methodological domain and item were graded for risk of bias using predefined criteria. Any discrepancies were resolved by consensus discussions (CW and GL) and, where necessary, by deferment to the third author (MM). No overall risk of bias judgement that summarised across domains was given due to the wide variation in assessment across domains within each tool. To improve the robustness of the synthesis and facilitate replicability, ${ }^{21}$ an overview of the domain definitions, items, effect measures, grades and criteria used in assessing the risk of bias is provided in online supplemental file 4.

\section{Synthesis methods}

In line with synthesis guidelines, ${ }^{22}$ a narrative approach was used to arrange the results into two categories: dermatology-specific and disease-specific tools psychosocial needs assessment tools. To ease identification of 


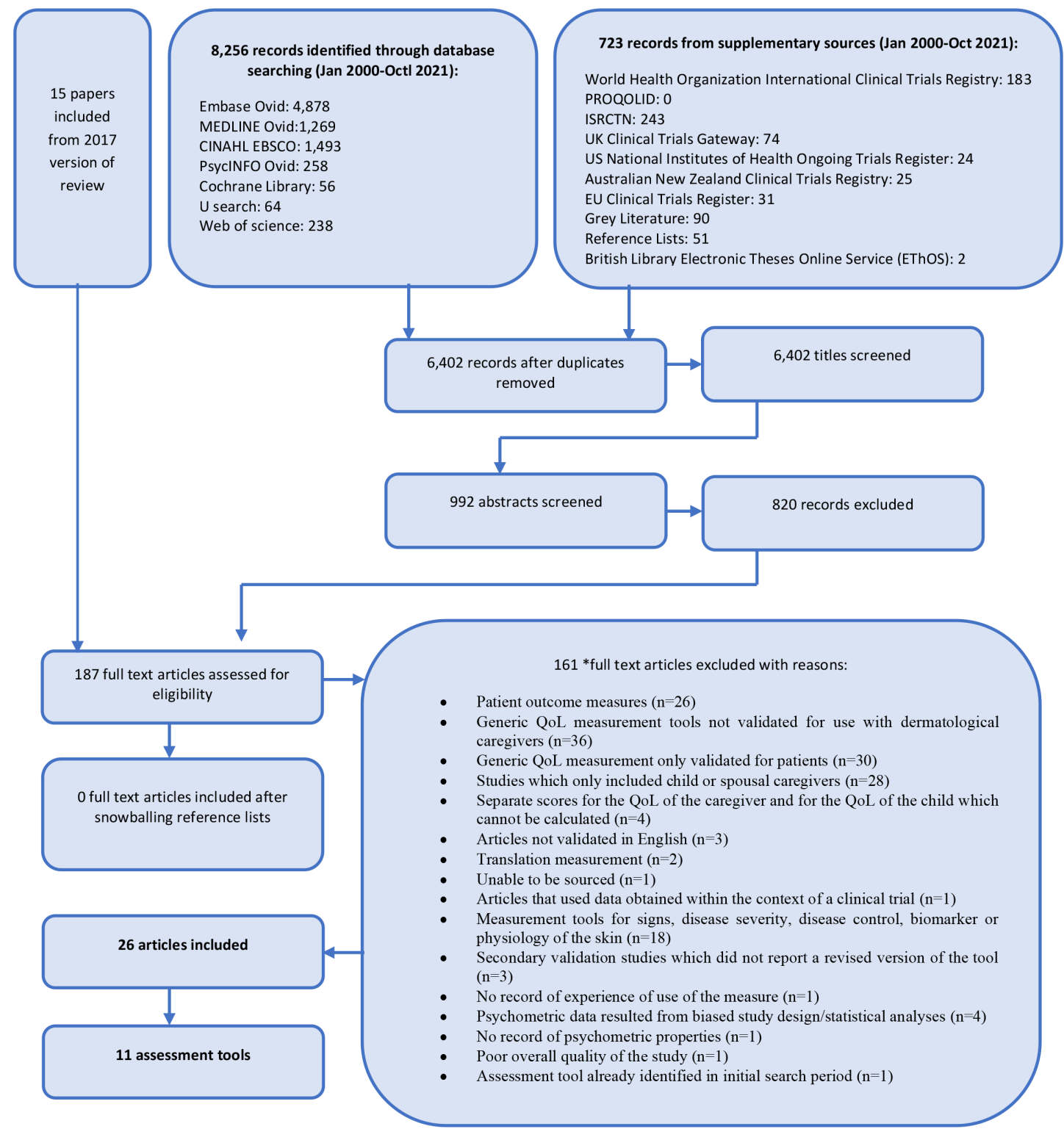

Figure 1 PRISMA, Preferred Reporting Items for Systematic Reviews and Meta-Analyses.; CINAHL, Cumulated Index to Nursing and Allied Health Literature; EBSCO, Elton B. Stephens Company; PsycINFO, Psychological Information Database; U Search, Ulster University Search; PROQOLID, Patient-Reported Outcome and Quality of Life Instruments Database; ISRCTN, International Standard Randomised Controlled Trials Number; UK, United Kingdom; US, United States; EU, European Union; QoL, Quality of Life.

variability between and within the included tools, results were also tabulated using the subheadings used in each of the three data extraction forms.

\section{Certainty assessment}

The robust search strategy was validated in MEDLINE when it successfully identified the one known systematic review ${ }^{19}$ as part of the search strategy development process (online supplemental file 1). Two authors (CW and GL) independently assessed the certainty of evidence by assessing risk of bias using a predefined checklist of criteria. ${ }^{20}$

\section{Ethics approval}

Informed consent was obtained from all participating members of the expert group associated with the research project

\section{Patient and public involvement (PPI)}

An international multidisciplinary expert group $(n=15)$, including affected adults, clinical psychologists, clinical nurse specialists, consultant dermatologists, health policy advisors and caregivers, was established at the outset of the project (September 2017). Anonymity remains protected due to their ongoing involvement in another follow-on study. The Guidance for Reporting Involvement of Patients and the Public Short Form checklist was used to improve the reporting of PPI in our study. ${ }^{23}$ PPI helped identify the research question, guide in terms of review design (search strategy, inclusion and exclusion criteria and data extraction subheadings) and improve the dissemination of findings (invitations to poster and orally 
present at international dermatology and psychology conferences).

\section{RESULTS}

This review identified 8979 records: 8256 records from database searching and 723 records from supplementary sources. After duplicates were removed (n=2577), 6402 records were available. Of the 6402 titles screened, 992 abstracts were screened, and 187 full-text articles were assessed for eligibility. This included 15 records identified from the one known systematic review. Of the 187 full-text articles assessed for eligibility, 161 records were excluded for reasons that met the exclusion criteria (PRISMA flow diagram; figure 1). No full-text records were included after snowballing reference lists (48 screened).

To improve transparency, summaries of the records identified during the initial and updated searches, for both databases and supplementary sources, are included in online supplemental files 3 and 5. PRISMA flow diagrams are included for both the initial search (1 January 2000-1 April 2020) (online supplemental file 5 (figure 1)) and updated search periods (1 April 2020-5 October 2020) (online supplemental file 5 (figure 2)) and provide a breakdown of the number of records identified for each database and supplementary source. The two full-text articles, identified in the updated search, were both excluded when assessed for eligibility. One record $^{24}$ contained psychometric data resulting from a biased study design and statistical analysis ("validity was established in a limited range of subjects', 'the parents that responded to the survey were all mothers', 'singleinstitution cross-sectional study in Japan targeting parents of first-time patients less than 7 years old'). The other record $^{25}$ identified the Family Dermatology Life Quality Index (FDLQI), which was already identified in the initial search.

The majority of existing, validated dermatological assessment tools identified were generic quality of life (QoL) tools and/or assess the patients' QoL. Of those tools validated for use among caregivers, most were either relevant to spouse/partner or depend on the caregiver to complete but are based on their child's perception of the disease (figure 1). Very few needs assessment tools were validated for use among caregivers of paediatric patients affected with dermatological disease. In summary, a total of 11 assessment tools were identified from the 26 articles included in this review. ${ }^{26-36}$ Ten disease-specific assessment tools were identified (ThePsoriasis Family Index (PFI15), ${ }^{26}$ Family Pso, ${ }^{27}$ Quality of life in Primary Caregivers of Children with Atopic Dermatitis (QPCAD), ${ }^{28}$ Childhood Atopic Dermatitis Impact Scale (CADIS), ${ }^{29}$ Parents' Index of Quality of Life in Atopic Dermatitis (PIQoL-AD), ${ }^{30}$ Dermatitis Family Impact (DFI), ${ }^{31}$ Parental Self-Efficacy with Eczema Care Index (PASECI), ${ }^{32}$ CareGiver Oncology Quality of Life (CarGOQoL), ${ }^{33}$ Epidermolysis Bullosa - Burden of Disease (EB-BoD) ${ }^{34}$ and Family Burden of Ichthyosi $(\mathrm{FBI})^{35}$ ) and one dermatology-specific assessment tool was identified $\left(\mathrm{FDLQ}^{36}\right)$. Table 1 provides a summary of study-specific information and includes the name of tool, country of origin, disease of affected patient, sample sizes and study setting. Table 2 summarises questionnaire-specific information under the subheadings outcome domains, subscales, number of items, recall period, scoring system and administration time. Table 3 provides an overview of the adequacy of the measurement properties of the included tools, including transferability, reliability, validity, structural and interpretability. Table 4 provides a graded risk of bias assessment (using the predefined criteria) of each methodological domain and item for each of the 11 tools.

\section{Disease-specific needs assessment tools}

The Psoriasis Family Index (PFI-15) ${ }^{26}$ is recommended for use alongside a dermatology-specific tool. As it is assessed on current time only, it does not rely on accurate recall. However, due to the small sample size, factor analysis could not be done, and there is a lack of comparison of PFI scores with other generic family QoL scales. In order to achieve its Cronbach's alpha value $(0.86)$, it was necessary to delete five items. It has a weaker focus on the emotional aspects of living with affected members. Those accompanying patients to the primary care centre and inpatients were not included in the creation of the PFI, which restricts the generalisation of the quantitative findings.

The Family $\mathrm{Pso}^{27}$ was created from interviews $(\mathrm{n}=95)$ with psoriasis patients and their family members. Three experts (no caregiver involvement) decided the generation items for piloting and item reduction. Other limitations include that a small sample was used in its testing and were predominantly female partners of the interviewees. Its advantages include that the wording is more focused on emotional aspects of caregiving as opposed to HR-QoL.

Four tools were found that assess the impact of atopic dermatitis on the family. The QPCAD ${ }^{28}$ has a 1-week recall and has been validated for use among primary caregivers of children with AD in the Japanese version only. Convergent validity requires further study, and only caregivers of mild and moderate patients from an urban area were included in the study.

The CADIS $^{29}$ is validated for use with both patients and parents of patients younger than 6 years. Rasch analysis reduced the tool to a 45 -item version, which is responsive to clinical change in $\mathrm{AD}$.

The Parents' Index of Quality of Life in Atopic Dermatitis (PIQoL-AD) ${ }^{30}$ assesses the impact of AD on caregivers of affected children, aged 8 years or younger. The PIQoL-AD adopts a dichotomous response system, which is less sensitive to subtle changes in HR-QoL and includes only items that consider the negative aspects of psychological well-being.

The $\mathrm{DFI}^{31}$ tool is the tool most widely reported in studies, having been used in over 750 clinical trials, although often at longer intervals despite being 
Table 1 Study-specific information relevant to included assessment tools

\begin{tabular}{|c|c|c|c|c|c|}
\hline $\begin{array}{l}\text { References } \\
\text { of included } \\
\text { publications } \\
\text { (first author, year, } \\
\text { reference) }\end{array}$ & $\begin{array}{l}\text { Country of } \\
\text { origin }\end{array}$ & $\begin{array}{l}\text { Disease of affected } \\
\text { patients }\end{array}$ & $\begin{array}{l}\text { Name of } \\
\text { measurement } \\
\text { instrument }\end{array}$ & Sample size (n) & Study setting \\
\hline Eghlileb et al (2009) & UK & Psoriasis & $\begin{array}{l}\text { Psoriasis Family Index } \\
\text { (PFI-15) }\end{array}$ & $\begin{array}{l}\text { Interviews } \\
\text { (unknown) }\end{array}$ & $\begin{array}{l}\text { Monocentric } \\
\text { Outpatient clinic }\end{array}$ \\
\hline $\begin{array}{l}\text { Mrowietz et al } \\
(2017)^{27}\end{array}$ & Germany & Psoriasis & Family Pso & $\begin{array}{l}\text { Interviews (14) } \\
\text { Piloting (96) } \\
\text { Validation (96) }\end{array}$ & $\begin{array}{l}\text { Monocentric } \\
\text { Outpatient clinic }\end{array}$ \\
\hline $\begin{array}{l}\text { Kondo-Endo et al } \\
(2009)^{28}\end{array}$ & Japan & $\begin{array}{l}\text { Atopic } \\
\text { dermatitis }\end{array}$ & $\begin{array}{l}\text { QoL in Primary } \\
\text { Caregivers of children } \\
\text { with Atopic Dermatitis }\end{array}$ & $\begin{array}{l}\text { Interviews } \\
\text { (unknown) } \\
\text { Pilot (33) } \\
\text { Validation (400) }\end{array}$ & $\begin{array}{l}\text { Monocentric } \\
\text { Outpatient clinic }\end{array}$ \\
\hline $\begin{array}{l}\text { Chamlin et al } \\
(2005)^{29}\end{array}$ & USA & $\begin{array}{l}\text { Atopic } \\
\text { dermatitis }\end{array}$ & $\begin{array}{l}\text { Childhood Atopic } \\
\text { Dermatitis } \\
\text { Impact Scale }\end{array}$ & $\begin{array}{l}\text { Interviews } \\
\text { (unknown) } \\
\text { Piloting (20) } \\
\text { Validation (300) }\end{array}$ & $\begin{array}{l}\text { Two dermatology } \\
\text { paediatric practices } \\
\text { (San Francisco and } \\
\text { Chicago) }\end{array}$ \\
\hline $\begin{array}{l}\text { McKenna et al } \\
(2005)^{30}\end{array}$ & $\begin{array}{l}\text { UK, Netherlands, } \\
\text { Italy, Spain, USA, } \\
\text { Switzerland, } \\
\text { Germany, France } \\
\text { (simultaneous } \\
\text { development) }\end{array}$ & $\begin{array}{l}\text { Atopic } \\
\text { dermatitis }\end{array}$ & $\begin{array}{l}\text { Parent's Index QoL - } \\
\text { Atopic Dermatitis }\end{array}$ & $\begin{array}{l}\text { Interviews }(65) \\
\text { Piloting (140 total) } \\
\text { Validation (ranged } \\
\text { between countries } \\
45-328)\end{array}$ & $\begin{array}{l}\text { Monocentric } \\
\text { Outpatient clinic }\end{array}$ \\
\hline Lawson et al (1998) & UK & Dermatitis & $\begin{array}{l}\text { Dermatitis Family } \\
\text { Impact }\end{array}$ & $\begin{array}{l}\text { Interviews (29) and } \\
\text { focus groups (10) } \\
\text { Piloting (14) } \\
\text { Validation (56) }\end{array}$ & $\begin{array}{l}\text { Monocentric } \\
\text { Outpatient clinic }\end{array}$ \\
\hline $\begin{array}{l}\text { References } \\
\text { of included } \\
\text { publications } \\
\text { (first author, year, } \\
\text { reference) }\end{array}$ & $\begin{array}{l}\text { Country of } \\
\text { origin }\end{array}$ & $\begin{array}{l}\text { Disease of affected } \\
\text { patients }\end{array}$ & $\begin{array}{l}\text { Name of } \\
\text { measurement } \\
\text { instrument }\end{array}$ & Sample size (n) & Study setting \\
\hline Minaya et al (2012) $)^{33}$ & France & Skin cancer & $\begin{array}{l}\text { CareGiver Oncology } \\
\text { Quality of Life }\end{array}$ & $\begin{array}{l}\text { Interviews (77) } \\
\text { Piloting (837) } \\
\text { Validation } \\
\text { (unknown) }\end{array}$ & $\begin{array}{l}\text { Monocentric } \\
\text { Outpatient clinic }\end{array}$ \\
\hline $\begin{array}{l}\text { Dufresne et al } \\
(2015)^{34}\end{array}$ & France & $\begin{array}{l}\text { Epidermolysis } \\
\text { bullosa }\end{array}$ & $\begin{array}{l}\text { Epidermolysis } \\
\text { Bullosa - Burden of } \\
\text { Disease }\end{array}$ & $\begin{array}{l}\text { Complaints (23) } \\
\text { informed item } \\
\text { generation } \\
\text { Piloting (Lionbridge } \\
\text { institution) } \\
\text { Validation (55) }\end{array}$ & $\begin{array}{l}\text { Monocentric } \\
\text { Outpatient clinic }\end{array}$ \\
\hline $\begin{array}{l}\text { Dufresne et al } \\
(2013)^{35}\end{array}$ & France & Ichthyosis & $\begin{array}{l}\text { Family Burden } \\
\text { Ichthyosis }\end{array}$ & $\begin{array}{l}\text { Interviews (94) } \\
\text { Piloting (42) } \\
\text { Validation (30) }\end{array}$ & $\begin{array}{l}\text { Monocentric } \\
\text { Outpatient clinic }\end{array}$ \\
\hline Basra et al (2008) & UK & $\begin{array}{l}\text { All - general } \\
\text { dermatology } \\
\text { instrument }\end{array}$ & $\begin{array}{l}\text { Family Dermatology } \\
\text { Life Quality } \\
\text { Index }\end{array}$ & $\begin{array}{l}\text { Interviews (50) } \\
\text { Piloting (20) } \\
\text { Validation (14) }\end{array}$ & $\begin{array}{l}\text { Monocentric } \\
\text { Outpatient clinic }\end{array}$ \\
\hline
\end{tabular}

QoL, quality of life.

validated for use with a 1-week recall period. As most of DFI studies are in secondary care hospitals, there exists the possibility of maximising the chances of the DFI scores showing significant improvements following an intervention. ${ }^{31}$ Dodington's review ${ }^{37}$ found that internal consistency and test-retest reliability was adequately demonstrated but highlighted that psychometric measures were less well established due to a lack 
Table 2 Questionnaire-specific information relevant to included assessment tools

\begin{tabular}{|c|c|c|c|c|c|c|}
\hline $\begin{array}{l}\text { Name of } \\
\text { measurement } \\
\text { instrument }\end{array}$ & $\begin{array}{l}\text { Outcome domains } \\
\text { measured }\end{array}$ & $\begin{array}{l}\text { Number of } \\
\text { items and } \\
\text { subscales }\end{array}$ & $\begin{array}{l}\text { Recall } \\
\text { period }\end{array}$ & Scoring system & $\begin{array}{l}\text { Respondent } \\
\text { feedback }\end{array}$ & $\begin{array}{l}\text { Admin mode } \\
\text { (time in minutes) }\end{array}$ \\
\hline $\begin{array}{l}\text { Psoriasis Family Index } \\
\text { (PFI-15) }^{26}\end{array}$ & $\begin{array}{l}\text { Social life, leisure activities, } \\
\text { sporting activities, people's } \\
\text { reactions, worry about future, } \\
\text { housework, relationships } \\
\text { treatment duration, clothing } \\
\text { shopping and sleep }\end{array}$ & 15 items & Now & 4-point scale (0-3) & $\begin{array}{l}\text { Brief in length. } \\
\text { Simple to administer, } \\
\text { score and interpret. } \\
\text { Weak evidence of } \\
\text { alternative forms. }\end{array}$ & $\begin{array}{l}\text { Self- administered } \\
\text { (2) }\end{array}$ \\
\hline Family $\mathrm{Pso}^{27}$ & $\begin{array}{l}\text { Emotional domain - } \\
\text { emotional impact. Social } \\
\text { domain -impact on daily } \\
\text { activities and work/school } \\
\text { and treatment. Leisure } \\
\text { domain - influence on leisure/ } \\
\text { personal relationships }\end{array}$ & 15 items & 1 month & $\begin{array}{l}\text { 5-point Likert format } \\
\text { (0-4) and 'Does not } \\
\text { apply' }\end{array}$ & $\begin{array}{l}\text { Brief in length. } \\
\text { Simple to administer, } \\
\text { score and interpret. } \\
\text { Weak evidence of } \\
\text { alternative forms. }\end{array}$ & $\begin{array}{l}\text { Self- administered } \\
\text { (3) }\end{array}$ \\
\hline $\begin{array}{l}\text { QoL in Primary } \\
\text { Caregivers of } \\
\text { children with Atopic } \\
\text { Dermatitis }^{28}\end{array}$ & $\begin{array}{l}\text { Achievement (3) } \\
\text { Worry (6) } \\
\text { Family cooperation (3) } \\
\text { Exhaustion (8) }\end{array}$ & 19 items & Past week & $\begin{array}{l}\text { 5-point scale (none } \\
\text { to extremely) }\end{array}$ & $\begin{array}{l}\text { Brief in length. } \\
\text { Moderate to administer, } \\
\text { score and interpret. } \\
\text { Conflicting evidence of } \\
\text { alternative forms. }\end{array}$ & $\begin{array}{l}\text { Self-report } \\
\text { (unknown) }\end{array}$ \\
\hline $\begin{array}{l}\text { Childhood Atopic } \\
\text { Dermatitis } \\
\text { Impact Scale }\end{array}$ & $\begin{array}{l}\text { Impact on family (three } \\
\text { domains) } \\
\text { Sleep and emotions } \\
\text { Family and social function }\end{array}$ & 45 items & 1 month & $\begin{array}{l}\text { 5-point scale (never to } \\
\text { all the time) }\end{array}$ & $\begin{array}{l}\text { Long in length and } \\
\text { problems of acceptability. } \\
\text { Moderate to administer, } \\
\text { score and interpret. } \\
\text { Absent evidence of } \\
\text { alternative forms. }\end{array}$ & $\begin{array}{l}\text { Self- administered } \\
\text { (6) }\end{array}$ \\
\hline $\begin{array}{l}\text { Parent's Index QoL - } \\
\text { Atopic Dermatitis }\end{array}$ & $\begin{array}{l}\text { One domain - needs that can } \\
\text { be influenced by a child with } \\
\text { a diagnosis of } A D \text {. }\end{array}$ & 28 items & Not reported & $\begin{array}{l}\text { 5-point scale (never to } \\
\text { all the time) }\end{array}$ & $\begin{array}{l}\text { Brief in length. } \\
\text { Simple to administer, } \\
\text { score and interpret. } \\
\text { Weak evidence of } \\
\text { alternative forms. }\end{array}$ & $\begin{array}{l}\text { Self- administered } \\
\text { (3) }\end{array}$ \\
\hline 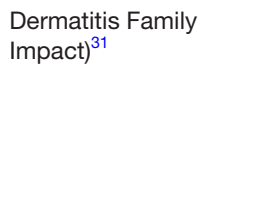 & $\begin{array}{l}\text { Personal relationships and } \\
\text { helping with treatment, } \\
\text { Food and feeding, sleep, } \\
\text { housework } \\
\text { shopping, financial, leisure } \\
\text { tiredness and emotional } \\
\text { distress }\end{array}$ & 10 items & 1 week & $\begin{array}{l}\text { 4-point scale (not at all, } \\
\text { a little, a lot, very much) }\end{array}$ & $\begin{array}{l}\text { Brief in length } \\
\text { Simple to administer, } \\
\text { score and interpret. } \\
\text { Weak evidence of } \\
\text { alternative forms. }\end{array}$ & $\begin{array}{l}\text { Self- administered } \\
\text { (unknown) }\end{array}$ \\
\hline $\begin{array}{l}\text { Parental Self-Efficacy } \\
\text { with } \\
\text { Eczema Care Index }\end{array}$ & $\begin{array}{l}\text { Managing medications } \\
\text { Managing eczema and } \\
\text { symptoms } \\
\text { Communication with } \\
\text { healthcare teams } \\
\text { Managing personal } \\
\text { challenges }\end{array}$ & $\begin{array}{l}29 \text { items } \\
\text { four } \\
\text { subscales }\end{array}$ & $\begin{array}{l}1 \text { week } \\
\text { preintervention } \\
\text { and } \\
4 \text { weeks } \\
\text { postintervention }\end{array}$ & 11-point Likert Scale & $\begin{array}{l}\text { Brief in length. } \\
\text { Simple to administer, } \\
\text { score and interpret. } \\
\text { Weak evidence of } \\
\text { alternative forms. }\end{array}$ & $\begin{array}{l}\text { Clinician } \\
\text { administered (3) }\end{array}$ \\
\hline $\begin{array}{l}\text { CareGiver Oncology } \\
\text { Quality of Life } \\
\text { questionnaire }^{33}\end{array}$ & $\begin{array}{l}\text { Psychological well-being, } \\
\text { burden, relationship with } \\
\text { healthcare, administration } \\
\text { and finances, coping, } \\
\text { physical well-being, self- } \\
\text { esteem, leisure time, } \\
\text { social support and private life }\end{array}$ & 29 items & 1 week & $\begin{array}{l}\text { 5-point Likert scale } \\
\text { (never/not at all, } \\
\text { rarely/a little, } \\
\text { sometimes/somewhat, } \\
\text { often/a lot, always/very } \\
\text { much) }\end{array}$ & $\begin{array}{l}\text { Brief in length. } \\
\text { Simple to administer, } \\
\text { score and interpret. } \\
\text { Weak evidence of } \\
\text { alternative forms. }\end{array}$ & $\begin{array}{l}\text { Self- administered } \\
\text { (3) }\end{array}$ \\
\hline $\begin{array}{l}\text { Epidermolysis } \\
\text { Bullosa - Burden of } \\
\text { Disease }^{34}\end{array}$ & $\begin{array}{l}\text { Economic and social impact } \\
(5) \\
\text { Family life (7) } \\
\text { Disease and treatment (5) } \\
\text { Child's life (3) }\end{array}$ & 20 items & Not stated & $\begin{array}{l}\text { 7-point scale } \\
\text { (always, very often, } \\
\text { often, sometimes, } \\
\text { rarely, never and not } \\
\text { applicable) }\end{array}$ & $\begin{array}{l}\text { Moderate to administer, } \\
\text { score and interpret. } \\
\text { Absent evidence of } \\
\text { alternative forms. Long in } \\
\text { length and problems of } \\
\text { acceptability. }\end{array}$ & $\begin{array}{l}\text { Self- administered } \\
\text { (unknown) }\end{array}$ \\
\hline $\begin{array}{l}\text { Family Burden } \\
\text { Ichthyosis }^{35}\end{array}$ & $\begin{array}{l}\text { Work and psychological } \\
\text { impact, daily life, pain, } \\
\text { familial and personal } \\
\text { relationships }\end{array}$ & 25 items & Not stated & $\begin{array}{l}\text { 4-point scale } \\
\text { (definitely yes, maybe, } \\
\text { definitely not and } \\
\text { I don't know) }\end{array}$ & $\begin{array}{l}\text { Long in length and } \\
\text { problems of acceptability. } \\
\text { Moderate to administer, } \\
\text { score and interpret. }\end{array}$ & $\begin{array}{l}\text { Self- administered } \\
\text { (3) }\end{array}$ \\
\hline $\begin{array}{l}\text { Family Dermatology } \\
\text { Life Quality } \\
\text { Index }\end{array}$ & $\begin{array}{l}\text { Housework and expenditure } \\
\text { Emotional and physical well- } \\
\text { being } \\
\text { Impact on study/job, social } \\
\text { life } \\
\text { burden of care, leisure } \\
\text { activities }\end{array}$ & 10 items & 1 month & $\begin{array}{l}\text { 4-point scale (not at all/ } \\
\text { not applicable, a little, } \\
\text { quite a lot and very } \\
\text { much) }\end{array}$ & $\begin{array}{l}\text { Brief in length. } \\
\text { Simple to administer, } \\
\text { score and interpret. } \\
\text { Weak evidence of } \\
\text { alternative forms. }\end{array}$ & $\begin{array}{l}\text { Self- administered } \\
\text { (3) }\end{array}$ \\
\hline
\end{tabular}

QoL, quality of care. 
Table 3 Adequacy of the measurement properties relevant to included assessment tools with excellent and good methodological quality

\begin{tabular}{|c|c|c|c|}
\hline $\begin{array}{l}\text { Name of } \\
\text { measurement } \\
\text { instrument }\end{array}$ & Transferability & Reliability & Validity \\
\hline $\begin{array}{l}\text { Psoriasis Family } \\
\text { Index } \\
(\text { PFI-15) }\end{array}$ & $\begin{array}{l}\text { Sometimes translated using } \\
\text { guidelines. } \\
\text { Never analysed in a cultural } \\
\text { equivalence study. }\end{array}$ & $\begin{array}{l}\text { IC: } 0.95>\text { Cronbach's } \\
\alpha>0.70 \text {. } \\
\text { Retest reliability: k or } \\
\text { ICC }>0.70\end{array}$ & $\begin{array}{l}\text { Conceptual - well balanced } \\
\text { domains. } \\
\text { Construct }>75 \% \text { results in } \\
\text { accordance with hypothesis. } \\
\text { Convergent - no information }\end{array}$ \\
\hline Family $\mathrm{Pso}^{27}$ & $\begin{array}{l}\text { Never translated using } \\
\text { guidelines. } \\
\text { Never analysed in a cultural } \\
\text { equivalence study. }\end{array}$ & $\begin{array}{l}\text { IC: } 0.95>\text { Cronbach's } \\
\alpha>0.70 \text {. } \\
\text { Retest reliability: } k \\
\text { or ICC not reported } \\
\text { or correlation } \\
\text { coefficient }<0.70 \text {. }\end{array}$ & $\begin{array}{l}\text { Conceptual - more focused on } \\
\text { objective/subjective domains. } \\
\text { Construct - no information. } \\
\text { Convergent }<0.70 \text {. }\end{array}$ \\
\hline
\end{tabular}

\begin{tabular}{|c|c|c|c|c|c|}
\hline $\begin{array}{l}\text { QoL in Primary } \\
\text { Caregivers of } \\
\text { children with } \\
\text { Atopic Dermatitis } \\
\end{array}$ & $\begin{array}{l}\text { Never translated using } \\
\text { guidelines. } \\
\text { Never analysed in a cultural } \\
\text { equivalence study. }\end{array}$ & $\begin{array}{l}\text { IC: } 0.95>\text { Cronbach's } \\
\alpha>0.70 \text {. } \\
\text { Retest reliability: } \mathrm{k} \text { or } \\
\text { ICC }>0.70 .\end{array}$ & $\begin{array}{l}\text { Conceptual: more focused on } \\
\text { objective/subjective domains. } \\
\text { Construct }<75 \% \text { results in } \\
\text { accordance with hypothesis. } \\
\text { Convergent }<0.70 \text {. }\end{array}$ & $\begin{array}{l}\text { Satisfactory response } \\
\text { to change in disease } \\
\text { severity. } \\
\text { Satisfactory test-retest } \\
\text { reliability. }\end{array}$ & $\begin{array}{l}\text { Norms: general nor } \\
\text { dermatology patients. } \\
\text { Categorisation: not } \\
\text { reported. } \\
\text { MCID: not reported. }\end{array}$ \\
\hline $\begin{array}{l}\text { Childhood Atopic } \\
\text { Dermatitis } \\
\text { Impact Scale }\end{array}$ & $\begin{array}{l}\text { Sometimes translated using } \\
\text { guidelines. } \\
\text { Never analysed in a cultural } \\
\text { equivalence study. }\end{array}$ & $\begin{array}{l}\text { IC: } 0.95>\text { Cronbach's } \\
\alpha>0.70 \text {. } \\
\text { Retest reliability: } \mathrm{k} \text { or } \\
\text { ICC }>0.70 .\end{array}$ & $\begin{array}{l}\text { Conceptual: well balanced } \\
\text { domains. } \\
\text { Construct }>75 \% \text { results in } \\
\text { accordance with hypothesis. } \\
\text { Convergent }<0.70 \text {. }\end{array}$ & $\begin{array}{l}\text { IRT. } \\
\text { Strong sensitivity to } \\
\text { detect changes. } \\
\text { Weak item bias. }\end{array}$ & $\begin{array}{l}\text { Norms: general nor } \\
\text { dermatology patients. } \\
\text { Categorisation: not } \\
\text { reported. } \\
\text { MCID: not reported. }\end{array}$ \\
\hline $\begin{array}{l}\text { Parent's Index } \\
\text { QoL - } \\
\text { Atopic Dermatitis }\end{array}$ & $\begin{array}{l}\text { Always translated using } \\
\text { guidelines. } \\
\text { Never analysed in a cultural } \\
\text { equivalence study. }\end{array}$ & $\begin{array}{l}\text { IC: } 0.95>\text { Cronbach's } \\
\alpha>0.70 \text {. } \\
\text { Retest reliability: } k \text { or } \\
\text { ICC }>0.70 .\end{array}$ & $\begin{array}{l}\text { Conceptual: more focused on } \\
\text { objective/subjective domains. } \\
\text { Construct }>75 \% \text { results in } \\
\text { accordance with hypothesis. } \\
\text { Convergent }<0.70 \text {. }\end{array}$ & $\begin{array}{l}\text { IRT. } \\
\text { Strong sensitivity to } \\
\text { detect changes item bias. } \\
\text { Strong item bias. }\end{array}$ & $\begin{array}{l}\text { Norms: general nor } \\
\text { dermatology patients. } \\
\text { Categorisation: not } \\
\text { reported. } \\
\text { MCID: known in } \\
\text { heterogeneous sample. }\end{array}$ \\
\hline $\begin{array}{l}\text { Dermatitis Family } \\
\text { Impact }^{31}\end{array}$ & $\begin{array}{l}\text { Always translated using } \\
\text { guidelines. } \\
\text { Sometimes analysed in a } \\
\text { cultural equivalence study. }\end{array}$ & $\begin{array}{l}\text { IC: } 0.95>\text { Cronbach's } \\
\alpha>0.70 \text {. } \\
\text { Retest reliability: } k \text { or } \\
\text { ICC }>0.70 .\end{array}$ & $\begin{array}{l}\text { Conceptual: well balanced. } \\
\text { Construct }<75 \% \text { results in } \\
\text { accordance with hypothesis. } \\
\text { Convergent }>0.70 \text {. }\end{array}$ & $\begin{array}{l}\text { No factor analysis or IRT. } \\
\text { Strong sensitivity to } \\
\text { detect changes. } \\
\text { Weak item bias. }\end{array}$ & $\begin{array}{l}\text { Norms: General nor } \\
\text { dermatology patients. } \\
\text { Categorisation: used } \\
\text { distribution-based } \\
\text { techniques. } \\
\text { MCID: not reported. }\end{array}$ \\
\hline $\begin{array}{l}\text { Parental Self- } \\
\text { Efficacy with } \\
\text { Eczema Care } \\
\text { Index }\end{array}$ & $\begin{array}{l}\text { Always translated using } \\
\text { guidelines. } \\
\text { Never analysed in a cultural } \\
\text { equivalence study. }\end{array}$ & $\begin{array}{l}\text { IC: } 0.95>\text { Cronbach's } \\
\alpha>0.70 \text {. } \\
\text { Retest reliability: } \mathrm{k} \text { or } \\
\text { ICC }>0.70 .\end{array}$ & $\begin{array}{l}\text { Conceptual: well balanced. } \\
\text { Construct }<75 \% \text { results in } \\
\text { accordance with hypothesis. } \\
\text { Convergent }>0.70 \text {. }\end{array}$ & $\begin{array}{l}\text { Factor analysis. } \\
\text { Satisfactory response } \\
\text { to change in disease } \\
\text { severity. } \\
\text { Weak item bias. }\end{array}$ & $\begin{array}{l}\text { Norms: general nor } \\
\text { dermatology patients } \\
\text { Categorisation: not } \\
\text { reported } \\
\text { MCID: not reported. }\end{array}$ \\
\hline $\begin{array}{l}\text { CareGiver } \\
\text { Oncology } \\
\text { Quality of Life } \\
\text { Questionnaire }^{33}\end{array}$ & $\begin{array}{l}\text { Sometimes translated using } \\
\text { guidelines. } \\
\text { Never analysed in a cultural } \\
\text { equivalence study. }\end{array}$ & $\begin{array}{l}\text { IC: } 0.95>\text { Cronbach's } \\
\alpha>0.70 \text {. } \\
\text { Retest reliability: } \mathrm{k} \text { or } \\
\text { ICC }<0.70 .\end{array}$ & $\begin{array}{l}\text { Conceptual: more focused on } \\
\text { objective/subjective domains. } \\
\text { Construct }<75 \% \text { results in } \\
\text { accordance with hypothesis. } \\
\text { Convergent }<0.70 \text {. }\end{array}$ & $\begin{array}{l}\text { Factor analysis. } \\
\text { Low/moderate sensitivity } \\
\text { to changes. } \\
\text { Weak item bias. }\end{array}$ & $\begin{array}{l}\text { Norms: general nor } \\
\text { dermatology patients. } \\
\text { Categorisation: used } \\
\text { distribution- based } \\
\text { techniques. } \\
\text { MCID: not reported. }\end{array}$ \\
\hline $\begin{array}{l}\text { Epidermolysis } \\
\text { Bullosa - Burden } \\
\text { of Disease }^{34}\end{array}$ & $\begin{array}{l}\text { Sometimes translated using } \\
\text { guidelines. } \\
\text { Never analysed in a cultural } \\
\text { equivalence study. }\end{array}$ & $\begin{array}{l}\text { IC: } 0.95>\text { Cronbach's } \\
\alpha>0.70 \text {. } \\
\text { Retest reliability: } \mathrm{k} \text { or } \\
\text { ICC }>0.70 .\end{array}$ & $\begin{array}{l}\text { Conceptual: well balanced. } \\
\text { Construct }<75 \% \text { results in } \\
\text { accordance with hypothesis. } \\
\text { Convergent }<0.70 \text {. }\end{array}$ & $\begin{array}{l}\text { Factor analysis. } \\
\text { Weak sensitivity to detect } \\
\text { changes. } \\
\text { Weak item bias. }\end{array}$ & $\begin{array}{l}\text { Norms: general nor } \\
\text { dermatology patients. } \\
\text { Categorisation: not } \\
\text { reported. } \\
\text { MCID: not reported. }\end{array}$ \\
\hline $\begin{array}{l}\text { Family Burden } \\
\text { Ichthyosis }^{35}\end{array}$ & $\begin{array}{l}\text { Sometimes translated using } \\
\text { guidelines. } \\
\text { Never analysed in a cultural } \\
\text { equivalence study. }\end{array}$ & $\begin{array}{l}\text { IC: } 0.95>\text { Cronbach's } \\
\alpha>0.70 \text {. } \\
\text { Retest reliability: } k \\
\text { or ICC not reported } \\
\text { or correlation } \\
\text { coefficient }<0.70 \text {. }\end{array}$ & $\begin{array}{l}\text { Conceptual: well balanced } \\
\text { Construct }<75 \% \text { results in } \\
\text { accordance with hypothesis. } \\
\text { Convergent }<0.70 \text {. }\end{array}$ & $\begin{array}{l}\text { No factor analysis or IRT. } \\
\text { Weak sensitivity to detect } \\
\text { changes. } \\
\text { Weak item bias. }\end{array}$ & $\begin{array}{l}\text { Norms: general nor } \\
\text { dermatology patients. } \\
\text { Categorisation: not } \\
\text { reported. } \\
\text { MCID: not reported. }\end{array}$ \\
\hline $\begin{array}{l}\text { Family } \\
\text { Dermatology Life } \\
\text { Quality } \\
\text { Index }^{36}\end{array}$ & $\begin{array}{l}\text { Always translated using } \\
\text { guidelines. } \\
\text { Never analysed in a cultural } \\
\text { equivalence study. }\end{array}$ & $\begin{array}{l}\text { IC: } 0.95>\text { Cronbach's } \\
\alpha>0.70 \text {. } \\
\text { Retest reliability: } \mathrm{k} \text { or } \\
\text { ICC }>0.70 \text {. }\end{array}$ & $\begin{array}{l}\text { Conceptual: well balanced } \\
\text { domains } \\
\text { Construct }>75 \% \text { results in } \\
\text { accordance with hypothesis. } \\
\text { Convergent }<0.70 \text {. }\end{array}$ & $\begin{array}{l}\text { Factor analysis. } \\
\text { Strong sensitivity to } \\
\text { detect changes. } \\
\text { Weak item bias. }\end{array}$ & $\begin{array}{l}\text { Norms: general nor } \\
\text { dermatology patients. } \\
\text { Categorisation: not } \\
\text { reported } \\
\text { MCID: not reported. }\end{array}$ \\
\hline
\end{tabular}

IRT, item response theory; IC, internal consistency; ICC, intraclass correlation coefficient; MCID, minimal clinically important difference.

of vigour in both the creation and validation processes. No valid score-banding descriptors of DFI score meanings are included, and no information to establish the
MCID of DFI score is available. ${ }^{31}$ No studies demonstrated dimensionality, factor structure or differential item functioning. 


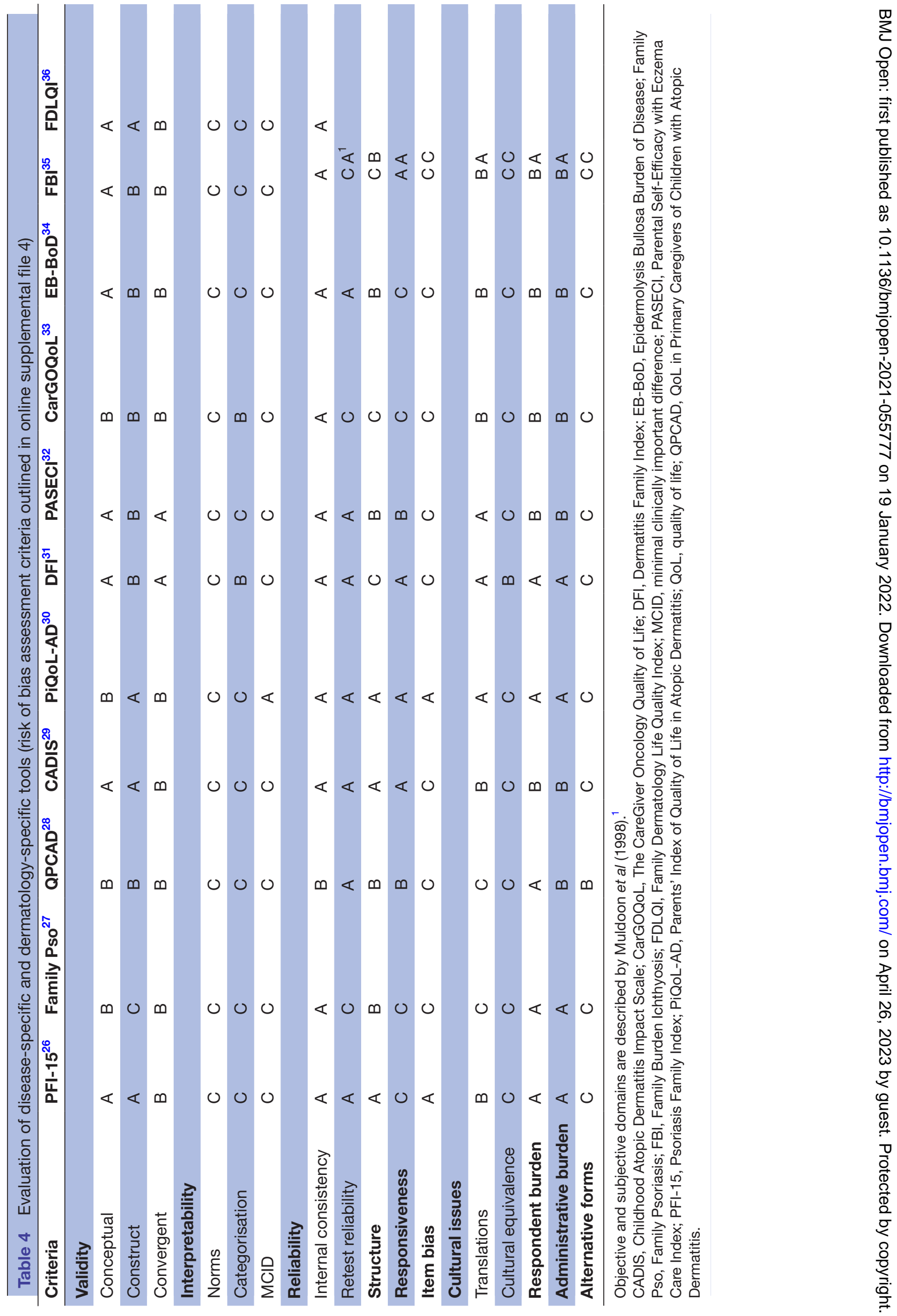


The final tool included in this review was the PASECI. ${ }^{32}$ It is a generalised self-efficacy scale focusing on the management of four subscales: medication, symptoms, personal challenges and communication with healthcare teams. It has a two-factor structure that considers the performance of routine management tasks and the management of child symptoms and behaviour. There was reliance on self-reported data, potentially affecting the fidelity of the results. More research is needed on banding and categorisation.

Validation of the CarGOQoL ${ }^{33}$ was carried out using dermatology experts other than caregivers. Several nonoptimal indicators of validity are indicated in table 4 .

The EB-BoD ${ }^{34}$ tool needed to remove nondiscriminatory items, such as frustration and guilt, from the original $\mathrm{FBI}^{38}$ during its creation. It requires further validation in larger EB patient and/or caregiver groups before being revalidated for use in other languages and cultures.

The $\mathrm{FBI}^{35}$ is the only validated disease-specific questionnaire that measures the concept of burden for ichthyosis caregivers. The monocentric study used parents and their affected children in the creation of verbatim using an unnamed French social assessment, which could not be accessed for this review. Selection bias was a possibility as $40 \%$ of participants cared for those affected by severe forms of ichthyosis (severity score 50 or greater). Limitations include that validation of the FBI was carried out using parents of children affected with only the severest forms of ichthyosis. Although itch is one of the significant challenges named by parents of children affected with ichthyosis (third most significant impact during the validation of the DFI), ${ }^{31}$ it does not feature as an item. Similarly, no items relate to pain in the finalised FBI. ${ }^{35}$ Verification of its psychometric properties, preferably in a multicentre study, is required. Caregiver feedback included that the finalised generation items were negatively phrased. The original French questionnaire has been linguistically and culturally adopted in Italy. ${ }^{38}$

\section{Dermatology-specific needs assessment tools}

The FDLQI ${ }^{36}$ is the most used dermatology-specific Health-Related Quality of Life (HR-QoL). The psychosocial impact loaded six items (emotional impact, physical well-being, impact on relationships, leisure, social life and people's reactions) and the physical impact loaded four items (burden, effect on job/study, household expenditure and housework). Fifty semistructured interviews took place that informed the items generated for testing during piloting. The feedback $(\mathrm{n}=59$ items) from these interviews has been termed 'the greater concept'. Piloting of the 19 items occurred with 20 parents or partners of those originally interviewed, potentially introducing bias. Limitations include that the life course of skin disease is not reflected in the FDLQI and that it depends on recall accuracy. Definitions, such as MID, and the meaning of FDLQI scores are missing and future research is required to show the unidimensionality of the tool. The FDLQI was not tested for responsiveness for clinical change in a hospital or intervention context. Several items cannot discriminate between inflammatory and uninflammatory groups.

One common theme that emerged was the variation in methodological rigour used in measuring informal dermatological caregiver needs. Using the risk of bias assessment, each of the reviewed tools indicated an incomplete psychometric overview meaning that the generalisability and interpretation of results remain limited. Each reviewed tool (11 of $11 ; 100 \%$ ) evaluated four or more psychometric properties. They do not comply with the OMERACT filter criteria and consequently are unable to be included in the development of a future Core Outcome Set (COS).$^{39}$

In terms of structure, five tools reported the use of factor analysis. ${ }^{27}$ 32-34 36 Three tools reported the use of the more recently developed item response theory (IRT) to determine psychometric properties. ${ }^{26} 2930$ Other tools neither reported factor analysis or IRT. ${ }^{28} 3135$ Apart from two tools reporting strong item bias, ${ }^{26}{ }^{30}$ the other nine tools ${ }^{27-2931-36}$ reported weak item bias. One tool ${ }^{31}$ reported the use of distribution-based categorisation techniques, but the other 10 tools did not report on categorisation. MCID was not reported for any tool other than one. ${ }^{30}$

In terms of reliability, all tools reported a high internal consistency (IC >0.95). Two tools did not report their retest reliability. ${ }^{27}$ One reported a weak retest reliability $^{33}$ (ICC $\left.<0.70\right)$, while the other eight tools reported a good retest reliability ${ }^{28-323436}$ (ICG $>0.70$ ). In terms of conceptual validity, four tools have less well-balanced domains. ${ }^{28-30} 33$ The other seven tools include well balanced domains..$^{26} 273132{ }^{34-36}$ No information is given regarding the construct validity for one tool. ${ }^{27}$ Five tools demonstrate that $<75 \%$ of results are in accordance with their hypothesis ${ }^{31-35}$ and five tools demonstrate that $>75 \%$ of results are in accordance with their hypothesis. ${ }^{26}$ 28-30 35 The majority of tools demonstrate poor convergent validity apart from two ${ }^{31} 32(>0.70)$. The PFI-15 provides no information on convergent validity. ${ }^{36}$ The other eight tools in this review show a convergent validity value of $<0.70 .{ }^{27-30} 33-36$

\section{DISCUSSION}

This is the first systematic review to address gaps in the existing evidence base around the identification of appropriate psychosocial needs assessment for caregivers of paediatric patients with dermatological conditions. This topic represents an emerging area for which there is a lack of up-to-date good quality synthesised evidence. With increasing numbers of paediatric patients of chronic skin disease being cared for by informal caregivers, often with limited medical training, key international multidisciplinary stakeholders (including clinicians, dermatological caregivers and policymakers) emphasised an urgent need to improve clinician awareness of existing needs assessment tools, to help them make informed evidence-based 
decisions relating to assessment. The need to promote caregiver health outcomes within day-to-day clinical practice has become even more significant during COVID-19, a period of enhanced social isolation and increased caregiver hypervigilance and burnout.

This review identified 11 psychosocial needs assessment tools validated for use among caregivers of paediatric patients with dermatological conditions. A narrative approach was used to arrange the reviewed tools into two groups: dermatology-specific and disease-specific tools. To ease identification of risk of bias, study variability and measurement properties between and within the included tools, results were additionally tabulated using the predefined subheadings on the data extraction forms.

Although skin disease may be characterised at times by unpredictable episodes in symptom severity, ${ }^{33} 34$ that requires similar systems of monitoring and integrated biopsychosocial support as other chronic conditions, ${ }^{5} 40$ our review highlights the lack of literature pertaining to the use of these assessment tools in healthcare settings. This review suggests that the mismatch between the recognised impact of caregiving for skin disease and the failure of practitioners to effectively engage with its management may be attributed to the biomedical model of assessment reflected in existing tools.

In contrast to the tools reviewed, ${ }^{26-36}$ which used measures of other constructs as a proxy for caregivers' need, it appears vital to directly assess informal dermatological caregivers' needs (at problem area and support level) and plan for how that knowledge will be used to help support these needs. ${ }^{41-43}$ Similarly, future assessments should use the scope of the International Classification of Functioning, Disability, and Health ${ }^{44}$ to inform their caregiver framework in terms of contextual factors and in terms of functioning and disability. Despite the recognised difficulty of assessing chronic pathologies by clinical or QoL aspects alone,${ }^{456}$ most tools identified in this review were generic QoL tools. The European Academy of Dermatology and Venereology Quality of Life Task Force, ${ }^{47}$ Cochrane Skin Centre of Evidence Based Dermatology ${ }^{48}$ and the Harmonising Outcome Measures for Eczema initiative ${ }^{39}$ reinforce that generic QoL assessments do not encompass the many factors that contribute to the psychosocial burden of skin disease ${ }^{49}$ and are not as sensitive, responsive or relevant to individual patients or their caregivers. ${ }^{50}$

We considered appropriate measurement tools to be theoretically driven, rigorously conceptualised with input from caregivers at each stage, consider disease life course, tested for validity and reliability and intended to assess caregiver needs in relevant settings. ${ }^{39} 414748$ Conceptual and theoretical work on dermatological caregivers' needs could have been relatively lacking because of the varying degree by which the tools were informed by caregiver experience, with minimal description of the questionnaire development process, absence of or exclusionary key definitions such as family, caregiver and domain and participants were not asked to clarify their relationship to the patient attending the outpatient clinics. Some of the tools only included items for the negative aspect of psychological well-being. ${ }^{31} 3536$

Healthcare teams require access to validated assessment tools that consider all dimensions along the care continuum and that do not use measures of other constructs as a proxy for caregivers' needs ${ }^{51}$ to provide culturally sensitive care. An international multicentric approach could best address variables including culture, demographics and disease severity. Although none of the reviewed assessment tools allow for the assessment of disease variables, including disease severity, we recommend that future needs assessment tools include disease parameters when designing their assessment framework. Dufresne $^{35}$ found that increased disease severity led to increased caregiver burden, suggesting that tools that assess factors relevant to clinical severity of disease could better inform the types of supports needed long term.

Future assessment should be practical and feasible for daily use within busy clinics. A self-reporting psychosocial needs assessment e-tool, developed to identify caregiver needs (at both problem and support level), could best serve to address non-clinical barriers to assessment, including lack of time, support staff and easy tools, to reduce the reported high rates of non-use of validated tools within daily practice. ${ }^{52}$ Research reinforces improved care recipient and caregiver outcomes ${ }^{41-44}$ when caregivers are facilitated to regularly selfreport perceived needs enabling clinicians to identify and/ or triage unmet psychosocial care needs.

\section{Strengths and limitations}

Strengths include a published protocol, a multidisciplinary expert group and health science librarian involved in the design of the review, a comprehensive literature search, information provision on study, questionnaire, measurement properties and risk of bias. This review also provides key recommendations for future research. Although time was needed to ensure that members were involved as equal partners in debates and decisions around key issues, benefits of PPI included having experts with lived experience who creatively contributed towards the methodology. Limitations included studies published in the English language between 2000 and 2021.

To enhance the chances of developing a truer set of outcome domains for improved COS uptake, future assessments should adopt a more thorough typology to assess the degree to which deficits in caregivers' needs are present and to develop transparent conceptual frameworks that include key definitions and that are built on a hybrid model using good quality caregiver frameworks alongside qualitative feedback from large and culturally diverse international cohorts of caregivers. ${ }^{53}$ With increased emphasis on e-healthcare, it seems both desirable and practical to conceptualise an accessible and solution-based model of future e-assessment that can address recognised healthcare challenges, including limited clinic time, poor caregiver identification and healthcare communication, ${ }^{53-58}$ allowing for timely identification and/or triage of unmet psychosocial needs by practitioners 
while strengthening a caregiver's sense of autonomy, coping ability and resilience. ${ }^{59}$ To inform the development of solution-focused assessment e-tools, it is important that research is also conducted into which supports are rated as most important by informal dermatological caregivers.

\section{CONCLUSION}

Although no gold standard tool exists for measuring the psychosocial needs of dermatological caregivers, this comprehensive review improves clinician awareness and knowledge of eleven validated psychosocial needs assessment tools for caregivers of paediatric patients with dermatological conditions. It is hoped that this review will inform the development of solution-based models of outcome assessment for improved dermatology care coordination. As dermatological caregiving research moves forward with significant public and private investment, rigorous measurement of caregivers' needs is essential for the development of social services, public policies and improved COS uptake. These findings have implications for clinical practice, service development and future research and reinforce that attitude towards caregivers is pivotal in developing assessment for the purpose of accessing supports and services.

\section{Twitter Carleen Walsh @CARLEENWALGAL}

Acknowledgements We would like to thank Ms Joan Atkinson, subject-specific librarian (Ulster University) for her assistance and guidance in devising a robust search strategy. We would also like to thank all members of the international multidisciplinary expert group for their guidance and expertise.

Contributors All authors and members of the expert group made substantial contribution to the design of the review. GL mentored and provided guidance to the review. All authors, together with a subject specific librarian (Joan Atkinson, Ulster University), created the robust search strategy and conducted the search. CW and GL screened articles, extracted and analysed data. Any discrepancies were resolved by deferment to the third author, ML; CW drafted the manuscript with valuable inputs from GL and $\mathrm{ML}$, and all authors reviewed drafts and approved the final version. CW acting as guarantor.

Funding Bamford Centre for Mental Health and Wellbeing (Ulster University). Award/Grant number is not applicable.

Competing interests Abstract was selected for paper presentation by the European Society for Dermatology \& Psychiatry Conference in June 2021. Abstract has also been selected for publication by the British Journal of Dermatology.

Patient consent for publication Not applicable.

Ethics approval Ethical approval for this review was obtained from University of Ulster Research Ethics Committee (Ref: REC/20/0004). Informed consent was obtained from all participating members of the expert group associated with the research project. Participants gave informed consent to participate in the study before taking part.

Provenance and peer review Not commissioned; externally peer reviewed.

Data availability statement All data relevant to the study are included in the article or uploaded as supplementary information. Not applicable.

Supplemental material This content has been supplied by the author(s). It has not been vetted by BMJ Publishing Group Limited (BMJ) and may not have been peer-reviewed. Any opinions or recommendations discussed are solely those of the author(s) and are not endorsed by BMJ. BMJ disclaims all liability and responsibility arising from any reliance placed on the content. Where the content includes any translated material, BMJ does not warrant the accuracy and reliability of the translations (including but not limited to local regulations, clinical guidelines, terminology, drug names and drug dosages), and is not responsible for any error and/or omissions arising from translation and adaptation or otherwise.
Open access This is an open access article distributed in accordance with the Creative Commons Attribution Non Commercial (CC BY-NC 4.0) license, which permits others to distribute, remix, adapt, build upon this work non-commercially, and license their derivative works on different terms, provided the original work is properly cited, appropriate credit is given, any changes made indicated, and the use is non-commercial. See: http://creativecommons.org/licenses/by-nc/4.0/.

\section{ORCID iDs}

Carleen Walsh http://orcid.org/0000-0002-7065-1066

Gerard Leavey http://orcid.org/0000-0001-8411-8919

\section{REFERENCES}

1 De Maeseneer H, Van Gysel D, De Schepper S, et al. Care for children with severe chronic skin diseases. Eur J Pediatr 2019;178:1095-103.

2 Manzoni APDdaS, Weber MB, Nagatomi ARdaS, et al. Assessing depression and anxiety in the caregivers of pediatric patients with chronic skin disorders. An Bras Dermatol 2013;88:894-9.

3 Karimkhani C, Dellavalle RP, Coffeng LE, et al. Global skin disease morbidity and mortality: an update from the global burden of disease study 2013. JAMA Dermatol 2017;153:406-12.

4 Flohr C, Hay R. Putting the burden of skin diseases on the global MAP. Br J Dermatol 2021;184:189-90.

5 Beattie P, Lewis-Jones M. A comparative study of impairment of quality of life in children with skin disease and children with other chronic childhood diseases. Br J Dermatol 2016;155:145-51.

6 Bickers DR, Lim HW, Margolis D, et al. The burden of skin diseases: 2004 a joint project of the American Academy of dermatology association and the Society for investigative dermatology. J Am Acad Dermatol 2006;55:490-500.

7 Lim HW, Collins SAB, Resneck JS, et al. The burden of skin disease in the United States. J Am Acad Dermatol 2017;76:958-72.

8 Basra MKA, Finlay AY. The family impact of skin diseases: the greater patient concept. Br J Dermatol 2007;156:929-37.

9 National Alliance for Caregiving. US national report on rare disease caregiving in America, 2018. Available: https://www.caregiving.org/ wpcontent/uploads/2018/02/NACRareDiseaseReport_February2018_WEB.pdf [Accessed 22 Jan 2021].

10 All-Party Parliamentary Group on Skin. Mental health and skin disease: a report of the All-Party parliamentary group on skin. 2020. Available: https://www.appgs.co.uk/publication/view/mental-healthand-skin-disease-2020/ [Accessed 01 Feb 2021].

11 National Institute for Health and Care Excellence (NICE). Common mental health problems: identification and pathways to care: clinical guidelines, 2011. Available: https://www.nice.org.uk/guidance/cg123 [Accessed 29 Dec 2020].

12 World Health Organisation (WHO). Evidence profile: caregiver support. integrated care for older people (ICOPE). guidelines on community-level interventions to manage declines in intrinsic capacity, 2017. Available: who.int/ageing/publications/guidelinesicope [Accessed 03 Jan 2021].

13 National Institute for health and care excellence (NICE). Atopic eczema in under 12S. Quality statement 3: psychological wellbeing and quality of life. Quality standard, 2013. Available: https:// www.nice.org.uk/guidance/qs44/chapter/Quality-statement-3Psychological-wellbeing-and-quality-of-life

14 National Institute for health and care excellence (NICE). Psoriasis: assessment and management. Clinical guideline. 153, 2007. https:// www.nice.org.uk/guidance/cg153

15 All Party Parliamentary Group on Skin (APPGS). Report on the enquiry into the impact of skin diseases on people's lives, 2003. Available: http://www.appgs.co.uk/publication/view/thepsychological-and-social-impact-of-skin-diseases-on-peoples-livesfinal-report-2013/ [Accessed 22 Feb 2021].

16 All Party Parliamentary Group on Skin (APPGS). Report on the psychological and social impact of skin diseases on people's lives, 2013. Available: https://www.appgs.co.uk/publication/view/thepsychological-and-social-impact-of-skin-diseases-on-peoples-livesfinal-report-2013/ [Accessed 15 Jan 2021].

17 Page MJ, McKenzie JE, Bossuyt PM, et al. The PRISMA 2020 statement: an updated guideline for reporting systematic reviews. BMJ 2021;372:n71.

18 Tong A, Flemming K, Mclnnes E, et al. Enhancing transparency in reporting the synthesis of qualitative research: ENTREQ. BMC Med Res Methodol 2012;12:181.

19 Sampogna F, Finlay AY, Salek SS, et al. Measuring the impact of dermatological conditions on family and caregivers: a review of 
dermatology-specific instruments. J Eur Acad Dermatol Venereol 2017;31:1429-39.

20 Both H, Essink-Bot M-L, Busschbach J, et al. Critical review of generic and dermatology-specific health-related quality of life instruments. J Invest Dermatol 2007;127:2726-39.

21 Page MJ, Moher D, Bossuyt PM, et al. PRISMA 2020 explanation and elaboration: updated guidance and exemplars for reporting systematic reviews. BMJ 2021;372:n160

22 Cochrane Consumers and Communication Review Group. Cochrane consumers and communication review group: data synthesis and analysis, 2013. Available: http://cccrg.cochrane.org [Accessed 10 Oct 2021].

23 Staniszewska S, Brett J, Simera I, et al. GRIPP2 reporting checklists: tools to improve reporting of patient and public involvement in research. BMJ 2017;358:j3453.

24 Sato H, Goto A, Murakami M, et al. Development of a pediatric dermatology screening tool based on two Parent-Reported skin symptoms: comparison of parental recognition and physician diagnosis of skin symptoms of infants and toddlers. J Prim Care Community Health 2020;11:1-7.

25 Żychowska M, Reich A, Maj J, et al. Impact of Childhood Psoriasis on Caregivers' Quality of Life, Measured with Family Dermatology Life Quality Index. Acta Derm Venereol 2020;100:adv00244.

26 Eghlileb AM, Basra MKA, Finlay AY. The psoriasis family index: preliminary results of validation of a quality of life instrument for family members of patients with psoriasis. Dermatology 2009;219:63-70.

27 Mrowietz U, Hartmann A, Weißmann W, et al. FamilyPso - a new questionnaire to assess the impact of psoriasis on partners and family of patients. J Eur Acad Dermatol Venereol 2017;31:127-34.

28 Kondo-Endo K, Ohashi Y, Nakagawa H, et al. Development and validation of a questionnaire measuring quality of life in primary caregivers of children with atopic dermatitis (QPCAD). Br J Dermatol 2009;161:617-25.

29 Chamlin SL, Cella D, Frieden IJ, et al. Development of the childhood atopic dermatitis impact scale: initial validation of a quality-of-life measure for young children with atopic dermatitis and their families. $J$ Invest Dermatol 2005;125:1106-11.

30 McKenna SP, Whalley D, Dewar AL, et al. International development of the parents' index of quality of life in atopic dermatitis (PIQoL-AD). Qual Life Res 2005;14:231-41.

31 Lawson V, Lewis-Jones MS, Finlay AY, et al. The family impact of childhood atopic dermatitis: the dermatitis family impact questionnaire. Br J Dermatol 1998;138:107-13.

32 Ersser SJ, Farasat $\mathrm{H}$, Jackson K, et al. Parental self-efficacy and the management of childhood atopic eczema: development and testing of a new clinical outcome measure. Br J Dermatol 2015;173:1479-85.

33 Minaya P, Baumstarck K, Berbis J, et al. The caregiver oncology quality of life questionnaire (CarGOQoL): development and validation of an instrument to measure the quality of life of the caregivers of patients with cancer. Eur J Cancer 2012;48:904-11.

34 Dufresne H, Hadj-Rabia S, Taieb C, et al. Development and validation of an epidermolysis bullosa family/parental burden score. $\mathrm{Br} J$ Dermatol 2015;173:1405-10.

35 Dufresne H, Hadj-Rabia S, Méni C, et al. Family burden in inherited ichthyosis: creation of a specific questionnaire. Orphanet J Rare Dis 2013;8:28.

36 Basra MKA, Edmunds O, Salek MS, et al. Measurement of family impact of skin disease: further validation of the family dermatology life quality index (FDLQI). J Eur Acad Dermatol Venereol 2008;22:813-21.

37 Dodington SR, Basra MKA, Finlay AY, et al. The dermatitis family impact questionnaire: a review of its measurement properties and clinical application. Br J Dermatol 2013;169:31-46.

38 El Hachem M, Abeni D, Diociaiuti A, et al. Italian translation, cultural adaptation, and pilot testing of a questionnaire to assess family burden in inherited ichthyoses. Ital J Pediatr 2019;45:26.

39 Schmitt J, Apfelbacher C, Spuls PI, et al. The Harmonizing outcome measures for eczema (home) roadmap: a methodological framework to develop core sets of outcome measurements in dermatology. $J$ Invest Dermatol 2015;135:24-30.

40 Vivar KL, Kruse L. The impact of pediatric skin disease on selfesteem. Int J Womens Dermatol 2018;4:27-31.
41 World Health Organisation WHO. Needs assessment. A healthcare guide, 2020. Available: https://www.who.int/health-cluster/resources/ publications/hc-guide/HC-Guide-chapter-10.pdf?ua=1 [Accessed 29 Dec 2020].

42 Public Health Information Services (PHIS) Toolkit. World Health organization, health cluster. 2018. Available: https://www.who.int/ healthcluster/resources/publications/PHIS-Toolkit/en/ [Accessed 23 Dec 2020]

43 European Council-Multi Purpose Grant (ERC-MPG) Consortium. Guidance and toolbox for the basic needs analysis: version 2, 2017. Available: https:// reliefweb.int/sites/reliefweb.int/files/resources/ basicneeds-assessment-guidanceoct17-3.pdf [Accessed 04 Jan 2021].

44 WHO Library Cataloguing-in-Publication Data. International classification of functioning, disability and health: ICF, 2001. Available: https://apps.who.int/iris/bitstream/handle/10665/42407/ 9241545429.pdf;jsessionid=01D148135C9BDFDDD7BFA524 EED8690B? sequence $=1$ [Accessed 15 Nov 2020].

45 de Vries M, Ouwendijk R, Kessels AG, et al. Comparison of generic and disease-specific questionnaires for the assessment of quality of life in patients with peripheral arterial disease. $J$ Vasc Surg 2005;41:261-8

46 Brown A, Page TE, Daley S, et al. Measuring the quality of life of family carers of people with dementia: development and validation of C-DEMQOL. Qual Life Res 2019;28:2299-310.

47 Chernyshov P, de Korte J, Tomas-Aragones L. EADV Quality of life task force. EADV taskforce's recommendations on measurement of health-related quality of life in paediatric dermatology. JEADV 2015;29:2306-16.

48 Collier A, Heilig L, Schilling L, et al. Cochrane skin group systematic reviews are more methodologically rigorous than other systematic reviews in dermatology. $\mathrm{Br} J$ Dermatol 2006;155:1230-5.

49 Toledano-Toledano F, Moral de la Rubia J. Factors associated with anxiety in family caregivers of children with chronic diseases. Biopsychosoc Med 2018;12:1-10.

50 Tan JD, Butow PN, Boyle FM, et al. A qualitative assessment of psychosocial impact, coping and adjustment in highrisk melanoma patients and caregivers. Melanoma Res 2014;24:252-60.

51 Penrod J, Hupcey JE, Shipley PZ, et al. A model of caregiving through the end of life: seeking normal. West $J$ Nurs Res 2012;34:174-93.

52 Chen V, Bellodi Schmidt F. Provider perceptions and practices for appearance-related psychosocial distress caused by dermatologic disease in children. Pediatr Dermatol 2021;38:1-6.

53 Williamson PR, Altman DG, Blazeby JM, et al. Developing core outcome sets for clinical trials: issues to consider. Trials 2012;13:132

54 Kaschowitz J, Brandt M. Health effects of informal caregiving across Europe: a longitudinal approach. Soc Sci Med 2017;173:72-80.

55 Finlay AY, Salek MS, Abeni D, et al. Why quality of life measurement is important in dermatology clinical practice: an expert-based opinion statement by the EADV Task force on quality of life. J Eur Acad Dermatol Venereol 2017;31:424-31.

56 Prinsen CAC, de Korte J, Augustin M, et al. Measurement of health-related quality of life in dermatological research and practice: outcome of the EADV Taskforce on quality of life. J Eur Acad Dermatol Venereol 2013;27:1195-203.

57 Weis A, Pohlmann S, Poss-Doering R, et al. Caregivers' role in using a personal electronic health record: a qualitative study of cancer patients and caregivers in Germany. BMC Med Inform Decis Mak 2020;20:158.

58 eMental HealthCullen K. State of the art opportunities for Ireland. work research centre, 2018. Available: https://www. mentalhealthreform.ie/wp-content/uploads/2018/10/eMental-HealthState-of-the-art-Opportunities-for-Ireland-Full-Report.pdf [Accessed 16 Jan 2021]

59 Holch P, Pini S, Henry AM, et al. eRAPID electronic patient selfreporting of Adverse-events: patient information and aDvice: a pilot study protocol in pelvic radiotherapy. Pilot Feasibility Stud 2018;4:1-15.

60 Astudillo I, Fuentes C, Herskovic V. Analízate: towards a platform to analyze activities and emotional states of informal caregivers. Proceedings 2018;2:1205-11. 